Pacific Journal of Mathematics

THE EXISTENCE OF WAVE OPERATORS FOR NONLINEAR EQUATING 


\title{
THE EXISTENCE OF WAVE OPERATORS FOR NONLINEAR EQUATIONS
}

\author{
A. R. BRoDSKY
}

In this paper, conditions will be found on nonlinear perturbations of the evolution equation with skew adjoint generator sufficient to guarantee the existence of nontrivial mild global solutions of the perturbed equation which converge to solutions of the unperturbed equation as $t \rightarrow+\infty$ or $-\infty$. These results are used to prove the existence of wave operators for certain semi-linear hyperbolic equations.

Let $H$ be a real separable Hilbert space and $A$ a skew adjoint linear operator on $H$. Denote the one parameter unitary group generated by $A$ by $e^{t A}$.

Let $K$ be a map from $R \times H \rightarrow H$ denoted by $K_{t}(u)$ for $u \in H$, $t \in R$. Assume also that $K_{t}(0)=0 \in H$ for all $t \in R$. The object of study in this paper will be functions $u: R \rightarrow H$ such that $K_{t}(u(t))$ is Bochner measurable and

$$
u(t)=e^{t A} u_{0}+\int_{0}^{t} e^{(t-s) \Delta} K_{s}(u(s)) d s
$$

where $u_{0}$ is some element of $H$. Note that it is assumed that $u$ is defined for all real $t$; i.e., it is a global solution of (1). An elementary sufficient condition that there exist such $u$ is that $K_{t}$ is globally Lipschitzian. That is, there exist $k$ such that

$$
\left\|K_{t}(u)-K_{t}(v)\right\| \leqq k\|u-v\|
$$

for all $t \in R$ and $u, v \in H$.

If $u(t)$ is strongly differentiable, is in $\mathscr{D}_{A}$ for all $t$ and satisfies (1) then

$$
\frac{d u}{d t}=A u+K_{t}(u) \quad u(0)=u_{0} .
$$

For this reason solutions of (1) are called global mild solution of (2). These are the only type of solutions this paper will be interested in.

The main question of this paper is whether there are solutions of (1) which behave for large positive or negative $t$ like $e^{t \Delta} v$ for some $v \in H$. If $\left\|K_{t}(u)-K_{t}(v)\right\| \leqq \sigma(t)\|u-v\|$ for all $u, v \in H$ and $\sigma$ bounded integrable then $u(t)$ exists for all $u_{0} \in H$ and behaves in this manner (Prop. 3). If $K$ does not depend on time, the results are not as straightforward. Propositions 4 and 5 give sufficient conditions on an element $v_{0} \in H$ that there exist a solution of (1) behaving as $t \rightarrow-\infty$ 
or $+\infty$ like $e^{t A} v_{0}$. These results are then applied to the question of existence of wave operators certain semi-linear hyperbolic equations which may be written in the form (1) or (2).

2. Wave operators. It is convenient to define a number of terms which will be useful later.

Definition. $\mathscr{W}_{-}\left(\mathscr{W}_{+}\right)$is a map from a subset $\mathscr{D}_{-}\left(\mathscr{D}_{+}\right)$of $H$ to a subset $\mathscr{R}_{-}\left(\mathscr{R}_{+}\right)$of $H$ defined as follows. $u_{0} \in \mathscr{D}_{-}$if $\lim _{t \rightarrow-\infty} e^{-t \Delta} u(t)$ exists where $u(t)$ is a solution of (1) with initial condition $u_{0}$.

$$
\mathscr{W}-u_{0}=\lim _{t \rightarrow-\infty} e^{-t \Delta} u(t) \text {. }
$$

$\mathscr{W}_{-}\left(\mathscr{W}_{+}\right)$is the backward (forward) wave operator.

REMARK. 1. The above objects depend on $A$ and $K_{t}$. If necessary they will be written $\mathscr{W}_{-}^{A, K}, \mathscr{D}_{-}^{A, K}$, etc.

2. All proofs will be for $\mathscr{W}_{-}, \mathscr{D}_{-}, \mathscr{R}_{-}$. Similar proofs with the obvious changes will work for $\mathscr{W}_{+}, \mathscr{D}_{+}, \mathscr{R}_{+}$.

3. If $u_{0} \in \mathscr{D}_{-}$and $w_{0}=\mathscr{W}_{-} u_{0} \in R_{-}$then $e^{t A} w_{0}$ satisfies (1) with $K_{t} \equiv 0$ and initial condition $w_{0}$. Moreover $\left\|e^{t A} w_{0}-u(t)\right\| \rightarrow 0$ as $t \rightarrow-\infty$.

Proposition 1. Let $w_{0}$ be in $H$. Suppose there exists a Bochner measurable function $v: R \rightarrow H$ such that

$$
\begin{gathered}
\int_{-\infty}^{0}\left\|K_{t}\left(e^{t A} v(t)\right)\right\| d t<\infty \\
v(t)=w_{0}+\int_{-\infty}^{t} e^{-s \Delta} K_{s}\left(e^{s A} v(s)\right) d s \quad t \leqq 0 .
\end{gathered}
$$

Then $w_{0} \in \mathscr{R}_{-}$and $v(0) \in \mathscr{D}_{-}$with $\mathscr{W}_{-} v(0)=w_{0}$.

Proof. Let $u(t)=e^{t \Delta} v(t)$. Then $u(0)=v(0)$. By $(\mathrm{b})$,

$$
\begin{aligned}
u(t) & =e^{t A} w_{0}+\int_{-\infty}^{t} e^{(t-s) \Delta} K_{s}(u(s)) d s \\
& =e^{t A} v(0)+\int_{0}^{t} e^{(t-s) \Delta} K_{s}(u(s)) d s
\end{aligned}
$$

$u(t)$ is a solution of (1) and with initial data $v(0)$, and $\left\|e^{-t A} u(t)-w_{0}\right\|=\left\|v(t)-w_{0}\right\| \leqq \int_{-\infty}^{t}\left\|K_{t}\left(e^{t A} v(t)\right)\right\| d t \rightarrow 0$ as $t \rightarrow-\infty$.

CoROllary 1. Let $u_{0}$ be in $H$ such there exists a solution $v(t)$ to 
(1) with initial data $u_{0}$. Then if $\int_{-\infty}^{0}\left\|K_{s}(u(s))\right\| d s<\infty, u_{0} \in \mathscr{D}_{-}$and

$$
\mathscr{W}_{-} u_{0}=u_{0}-\int_{-\infty}^{0} e^{-s A} K_{s}(u(s)) d s
$$

Proposition 2. If $K_{t}$ is Lipschitzian with constant $k$ and $u_{0}$ and $v_{0}$ are $\mathscr{D}_{-}$with $\mathscr{W}_{-} u_{0}=\mathscr{W}_{-} v_{0}$ then $u_{0}=v_{0}$ if

$$
\int_{-\infty}^{s}\|u(t)-v(t)\| d t \leqq \gamma e^{c s} \quad s \leqq 0, c>k
$$

and $u(t)$ and $v(t)$ satisfy the hypothesis of Corollary 1 .

Proof.

$$
\begin{aligned}
\|u(t)-v(t)\| & \leqq \int_{-\infty}^{t}\left\|K_{s}(u(s))-K_{s}(v(s))\right\| d s \\
& \leqq k \int_{-\infty}^{t}\|u(s)-v(s)\| d s
\end{aligned}
$$

Let $\sigma(t)=\|u(t)-v(t)\|$ and

$$
\varphi(t)=\int_{-\infty}^{t}\|u(s)-v(s)\| d s \leqq \gamma e^{c t}
$$

$\varphi(t)$ is absolutely continuous and $\varphi^{\prime}=\sigma$.

$$
\begin{aligned}
& \therefore \varphi^{\prime} \leqq k \varphi \\
& \therefore \quad \frac{d}{d t}\left(\varphi e^{-k t}\right) \leqq 0 \\
& \therefore \int_{T}^{t} \frac{d}{d t}\left(\varphi e^{-k t}\right) \leqq 0 \quad t \geqq T \\
& \text { i.e. } \varphi(t) e^{-k t} \leqq \varphi(T) e^{-k T} \leqq \gamma e^{(c-k) T} \rightarrow 0 \text { as } T \rightarrow-\infty \text {. } \\
& \therefore \varphi(t) e^{-k t} \leqq 0 . \quad t>-\infty \\
& \therefore \varphi(0)=0 \Longrightarrow \sigma(0)
\end{aligned}
$$

The following classical result will be helpful.

LEMMA 1. Let $\rho, \sigma, \varepsilon$ be positive real valued measurable functions, $\rho$ and $\varepsilon$ bounded and $\sigma$ integrable such that

$$
\rho(t) \leqq \varepsilon(t)+\int_{-\infty}^{t} \sigma(s) \rho(s) d s
$$

Then

$$
\rho(t) \leqq \varepsilon(t)+\exp \left(\int_{-\infty}^{t} \sigma(s) d s\right) \int_{-\infty}^{t} \varepsilon(s) \sigma(s) \exp \left(\int_{-\infty}^{s} \sigma(\alpha) d \alpha\right) d s
$$


The following is a result of Segal [2] and Strauss [6]. For $H=E^{n}$, this result is classical.

Proposition 3. If $\left\|K_{t}(u)-K_{t}(v)\right\| \leqq \sigma(t)\|u-v\|$ where $\sigma(t)$ is bounded integrable then $\mathscr{D}_{-}=\mathscr{R}_{-}=H$, $\mathscr{Y}_{-}$is one-one, Lipschitz continuous with a Lipschitz continuous inverse.

Proof. Let $u_{0} \subset H$. Let $u(t)$ be the solution of (1) with data $u_{0}$. Thus

$$
u(t)=e^{t A} u_{v}+\int_{0}^{t} e^{(t-s) A} K_{s}(u(s)) d s \quad t \leqq 0
$$

Thus

$$
\|u(t)\| \leqq\left\|u_{0}\right\|+\int_{t}^{0} \sigma(s)\|u(s)\| d s .
$$

Then by a classical result,

$$
\begin{aligned}
& \|u(t)\| \leqq\left\|u_{0}\right\|\left(1+c e^{c}\right) \text { where } c=\int_{-\infty}^{0} \sigma(t) d t . \\
\therefore \quad & \int_{-\infty}^{0}\left\|K_{s}(u(s))\right\| d s \leqq \int_{-\infty}^{0} \sigma(s)\|u(s)\| d s<c\left(1+c e^{c}\right)\left\|u_{0}\right\| .
\end{aligned}
$$

Thus $u_{0} \in \mathscr{D}_{-}$by Corollary 1. $\therefore \quad \mathscr{D}_{-}=H$. Let $u_{0}$ and $v_{0}$ be in $H$. Let $u(t)$ and $v(t)$ be the corresponding solutions. Then

$$
\begin{array}{ll}
\|u(t)-v(t)\| \leqq\left\|u_{0}-v_{0}\right\|+\int_{t}^{0} \sigma(s)\|u(s)-v(s)\| d s & t \leqq 0 . \\
\therefore \quad\|u(t)-v(t)\| \leqq\left\|u_{0}-v_{0}\right\|\left(1+c e^{c}\right) & \forall t \leqq 0 \\
\therefore \quad\left\|\mathscr{V} \_u_{0}-\mathscr{W} \_v_{0}\right\| \leqq\left\|u_{0}-v_{0}\right\|\left(1+c e^{c}\right) . &
\end{array}
$$

Thus $\mathscr{W} \_$is Lipschitz continuous. Now suppose $\mathscr{W}_{-} u_{0}=\mathscr{V}_{-} v_{0}$.

$$
\begin{aligned}
& e^{-t A} u(t)=u_{0}+\int_{0}^{t} e^{-s A} K_{s}(u(s)) d s . \\
& e^{-t A} v(t)=v_{0}+\int_{0}^{t} e^{-s A} K_{s}(v(s)) d s .
\end{aligned}
$$

Similarly,

$$
\begin{aligned}
& e^{-t A} u(t)=e^{-T A} u(T)+\int_{T}^{t} e^{(t-s) A} K_{s}(u(s)) d s \\
& e^{-t A} v(t)=e^{-T A} v(T)+\int_{T}^{t} e^{(t-s) A} K_{s}(v(s)) d s
\end{aligned}
$$

Therefore 


$$
\begin{aligned}
& \left\|e^{-t A} u(t)-e^{t A} v(t)\right\|=\|u(t)-v(t)\| \leqq\|u(T)-v(T)\| \\
& \quad+\int_{T}^{t} \sigma(s)\|u(s)-v(s)\| d s \quad 0 \geqq t \geqq T \\
& \|u(t)-v(t)\| \leqq\|u(T)-v(T)\|+\int_{-\infty}^{t} \sigma(s)\|v(s)-v(s)\| d s
\end{aligned}
$$

By Lemma 1,

$$
\|u(t)-v(t)\| \leqq\|u(T)-v(T)\|\left(1+c e^{c}\right) \quad 0 \geqq t \geqq T .
$$

But $\|u(T)-v(T)\| \rightarrow 0$ as $T \rightarrow-\infty$.

$\therefore\|u(t)-v(t)\|=0 \quad \forall t \leqq 0$. In particular for $t=0$.

$\therefore u_{0}=v_{0}$.

Let $T \leqq 0$ be such that $\int_{-\infty}^{T} \sigma(s) d s=c^{\prime}<1$. Let $u_{0} \in H$. Define recursively

$$
u_{n}(t)=u_{0}+\int_{-\infty}^{t} e^{-s A} K_{s}\left(e^{s A} u_{n-1}(s)\right) d s . \quad t \leqq T .
$$

Claim (a) $\left\|u_{n}(t)-u_{n-1}(t)\right\| \leqq c^{\prime n}\left\|u_{0}\right\|$

$$
t \leqq T
$$

(b) $\left\|u_{n}(t)\right\| \leqq\left\|u_{0}\right\| \sum_{k=0}^{n} c^{\prime n}$

$t \leqq T$.

These are proved by induction. Some care must be taken since $u_{n+1}$ is not defined until (b) is proved for $u_{n}$.

It follows that there exists a measurable $v(t)$ such that $u_{n}(t) \rightarrow v(t)$ uniformly on $(-\infty, T]$. Also, $\|v(t)\| \leqq\left\|u_{0}\right\| /\left(1-c^{\prime}\right)$ for $t \leqq T$.

It's trivial show there a unique $w(t): R \rightarrow H$ satisfying

$$
w(t)=e^{t A} v(T)+\int_{T}^{t} e^{(t-s) \wedge} K_{s}(w(s)) d s .
$$

For $t<T, e^{-t A} w(t)=v(t)$. Let $\widetilde{v}(t)=e^{-t A} w(t)$. $\widetilde{v}(t)$ satisfies the hypothesis of Proposition 1. $\therefore u_{0} \in \mathscr{R}_{-} . \therefore \mathscr{R}_{-}=H$. It follows from (3) that

$$
\begin{aligned}
& \|u(t)-v(t)\| \leqq\left\|\mathscr{W}-u_{0}-\mathscr{W}-v_{0}\right\|\left(1+c e^{c}\right) \quad t \leqq 0 \\
\therefore \quad & \left\|u_{0}-v_{0}\right\| \leqq\left\|\mathscr{W}-u_{0}-\mathscr{W} v_{0}\right\|\left(1+c e^{c}\right) \text { for } u_{0} \& v_{0} \in H . \\
\therefore \quad & \left\|\mathscr{W}_{-}^{-1} u_{0}-\mathscr{W}_{-}^{-1} v_{0}\right\| \leqq\left\|u_{0}-v_{0}\right\|\left(1+c e^{c}\right) .
\end{aligned}
$$

Corollary 2. $S=\mathscr{W}_{+} \mathscr{W}_{-}^{-1}$ exists and is Lipschitz continuous.

Proposition 4. Let $\beta \in H$. Let $K$ independent of $t$ and Lipschitz cont. with Lipschitz constant $k$. If $\left\|K\left(e^{t A} \beta\right)\right\| \leqq d e^{c t}$ for $t \leqq T$ (some fixed $T$ ) and $c>k$, then $\beta \in \mathscr{R}_{-}$and in fact there is a solution $u(t)$ such that 
$(*)$

$$
\left\|e^{-t A} u(t)-\beta\right\| \leqq \frac{d}{c-k} e^{c t} \quad t \leqq T .
$$

Moreover, $u(t)$ is unique among solutions satisfying *.

Proof. The proof is a Picard iteration. Because of the technical details, a proof is included. Let $v_{0}(t)=\beta$.

$$
v_{n}(t)=\beta+\int_{-\infty}^{t} e^{-s A} K\left(e^{s A} v_{n-1}(s)\right) d s \quad t \leqq T .
$$

Claim
(a) $\left\|v_{n}(t)-v_{n-1}(t)\right\| \leqq \frac{d k^{n-1}}{c^{n}} e^{c t}$
$t \leqq T$
(b) $\int_{-\infty}^{t}\left\|K\left(e^{s A} v_{n}(s)\right)\right\| d s \leqq \frac{d}{k} e^{c t} \sum_{j=1}^{n-1}\left(\frac{k}{c}\right)^{j}$
$t \leqq T$$$
\left\|v_{1}(t)-\beta\right\| \leqq \int_{-\infty}^{t}\left\|K\left(e^{s A} \beta\right)\right\| d s \leqq \frac{d}{c} e^{c t} .
$$

$\therefore \quad$ (a) holds for $n=1$. Moreover

$$
\begin{aligned}
& \int_{-\infty}^{t} \| K\left(e ^ { s A } v _ { 1 } ( s ) \| d s \leqq \int _ { - \infty } ^ { t } \| K \left(e^{s A} v_{1}(s)-K\left(e^{s \Lambda} \beta\right) \| d s\right.\right. \\
&+\int_{-\infty}^{t}\left\|K\left(e^{s A} \beta\right)\right\| d s \leqq \frac{d}{k} e^{c t}\left(\frac{R}{c}+\frac{R^{2}}{c^{2}}\right) \quad t \leqq T
\end{aligned}
$$

$\therefore$ (b) holds for $n=1$.

Assume $v_{n-1}(t)$ exists and satisfies (b). Thus $v_{n}(t)$ exists.

$$
\int_{-\infty}^{t}\left\|K\left(e^{s A} v_{n}(s)\right)\right\| d s \leqq \int_{-\infty}^{t}\left\|v_{n}-\beta\right\| d s+\frac{d}{c} e^{c t} \quad t \leqq T .
$$

But

$$
\begin{array}{rlrl}
\left\|v_{n}-\beta\right\| \leqq \int_{-\infty}^{t}\left\|K\left(e^{s A} v_{n-1}(s)\right)\right\| d s \leqq \frac{d}{k} e^{c t} \sum_{j=1}^{n}\left(\frac{k}{c}\right)^{j} & t \leqq T \\
\therefore \int_{-\infty}^{t}\left\|K\left(e^{s A} v_{n}(s)\right)\right\| d s \leqq \frac{d}{k} e^{c t}+\frac{d}{c} e^{c t} \sum_{j=1}^{n}\left(\frac{k}{c}\right)^{j} & \\
=\frac{d}{k} e^{c t} \sum_{j=1}^{n+1}\left(\frac{k}{c}\right)^{j} & t \leqq T \\
\left\|v_{n}(t)-v_{n-1}(t)\right\| \leqq k \int_{-\infty}^{t}\left\|v_{n-1}(s)-v_{n-2}(s)\right\| d s & \\
& \leqq \frac{d k^{n-1}}{c^{n}} e^{c t} & t \leqq T .
\end{array}
$$

Thus $v_{n}(t)$ exist for all $n$ and $t \leqq T$ and satisfy (a) and (b). Thus $v_{n}(t) \rightarrow v(t)$ uniformly on $(-\infty, T)$. In fact, 


$$
\left\|v_{n}(t)-v(t)\right\| \leqq\left(\frac{k}{c}\right)^{n} \frac{d c^{c t}}{k-c}
$$

$v(t)$ satisfies

$$
v(t)=\beta-\int_{-\infty}^{t} e^{-s A} K\left(e^{s A} v(s)\right) d s
$$

If $T \geqq 0$ then $v(0)$ is already defined and by Theorem $1, \beta \in R$. If $T<0$, let $w(t)$ satisfy

$$
w(t)=e^{t A} v(T)+\int_{T}^{t} e^{(t-s) A} K(w(s)) d s
$$

$w(t)$ exists for all $t$ and $w(t)=e^{t A} v(t)$ for $t \leqq T$.

If $\widetilde{v}(t)=e^{-t A} w(t)$ then $\widetilde{v}(t)$ satisfies the hypothesis of Proposition 1 . Thus $\beta \in \mathscr{R}_{\text {_. }} w(t)$ is our sought after $u(t)$. For $t \leqq T$,

$$
\begin{aligned}
\left\|e^{-t A} w(t)-\beta\right\| & =\|v(t)-\beta\| \leqq\left\|v(t)-v_{n}(t)\right\|+\left\|v_{n}(t)-\beta\right\| \\
& \leqq\left(\frac{k}{c}\right)^{n} \frac{d e^{c t}}{c-k}+\frac{d e^{c t}}{k} \sum_{j=1}^{n}\left(\frac{k}{c}\right)^{j}=\frac{d e^{c t}}{c-k}
\end{aligned}
$$

Suppose $\mathscr{W}_{-} z(0)=\beta\left\|z(t)-e^{t A} \beta\right\| \leqq \gamma^{\prime} e^{c^{\prime} t} c^{\prime}>k$ for $t \leqq T^{\prime}$.

Then

$$
\|z(t)-w(t)\| \leqq \gamma e^{c^{\prime} t}+\frac{d}{c-k} e^{c t} \quad t<\min \left(T, T^{\prime}\right)
$$

Then $z(0)=w(0)$ by Proposition 2 .

Proposition 5. Let

$$
k(s)=\sup _{\|u\|,\|v\| \leqq r} \frac{\|K(u)-K(v)\|}{\|u-v\|} .
$$

Assume $k(s)$ is bounded on compact subsets of $R^{+}$. Assume also that for any $u_{0} \in H$, there is a solution of (1) with data $u_{0}$. Let $\beta \in H$ and assume $\left\|K\left(e^{t A} \beta\right)\right\| \leqq \gamma e^{c t}$ for $t \leqq T^{\prime \prime}$ and $c>k(2\|\beta\|)$. Then $\beta \in \mathscr{R}_{\text {. }}$.

Proof. Let $T^{\prime}$ be such that if $t \leqq T^{\prime}, \gamma e^{c t} \leqq(c-k)\|\beta\|, k=$ $k(2\|\beta\|)$. Let $T=\min \left(T^{\prime}, T^{\prime \prime}\right)$. Let $v_{0}(t)=\beta$. Let

$$
v_{1}(t)=\beta+\int_{-\infty}^{t} e^{-s A} K\left(e^{s A} \beta\right) d s \quad t \leqq T
$$

$$
\begin{array}{ll}
\text { (i) }\left\|v_{1}(t)-\beta\right\| \leqq(\gamma / c) e^{c t} & t \leqq T \\
\text { (ii) }\left\|v_{1}(t)\right\| \leqq\|\beta\|+(\gamma / c) e^{c t} \leqq(2-k / c)\|\beta\| & t \leqq T .
\end{array}
$$

It follows that $v_{2}(t)$ is defined as is Proposition 4. 
Claim $\left\|v_{n}(t)\right\| \leqq\left(2-\left(\frac{k}{c}\right)^{n}\right)\|\beta\| . \quad t \leqq T$ True to $n=1$

$$
\left\|v_{n}(t)-v_{n-1}(t)\right\| \leqq \int_{-\infty}^{t}\left\|K\left(e^{s A} v_{n-1}(s)\right)-K\left(e^{s A} v_{n-2}(s)\right)\right\| d s \quad t \leqq T \text {. }
$$

By induction assumption, $\left\|v_{n-1}(s)\right\|$ and $\left\|v_{n-2}(s)\right\|$ are both less than $2\|\beta\|$ for $s \leqq T$.

$$
\left.\therefore \quad\left\|v_{n}(t)-v_{n-1}(t)\right\| \leqq k \int_{-\infty}^{t} \| v_{n-1}(s)-v_{n-2}(s)\right) \| d s .
$$

But $\left\|v_{0}\right\|,\left\|v_{1}\right\| \cdots\left\|v_{n-1}\right\| \leqq 2\|\beta\|$.

$\therefore$ for $j<n$,

$$
\left\|v_{j}(t)-v_{j-1}(t)\right\| \leqq k \int_{-\infty}^{t}\left\|v_{j-1}(s)-v_{j-2}(s)\right\| d s \leqq \frac{\gamma k^{j-1}}{c^{j}} e^{c t}
$$

by induction.

$$
\begin{aligned}
& \therefore \quad\left\|v_{n}(t)-v_{n-1}(t)\right\| \leqq \frac{\gamma k^{n-1}}{c^{n}} e^{c t} \\
& \therefore \quad\left\|v_{n}(t)\right\| \leqq\left\|v_{n-1}(t)\right\|+\frac{\gamma k^{n-1}}{c^{n}} e^{c t} \leqq\left(2-\left(\frac{k}{c}\right)^{n}\right)\|\beta\| .
\end{aligned}
$$

Now proceed as in Proposition 4.

3. Nonlinear wave equation. Consider the partial differential equations

$$
\begin{aligned}
& \square u=0 \\
& \square u=q F(u)
\end{aligned}
$$$$
\begin{aligned}
& u(0)=r(\vec{x}) \quad u_{t}(0)=s(\vec{x}) \\
& u(0)=r(\vec{x}) \quad u_{t}(0)=s(\vec{x})
\end{aligned}
$$

where $\square=\sum_{\jmath_{1}=1}^{3}\left(\partial^{2} / \partial x_{j}^{2}\right)-\left(\partial^{2} / \partial t^{2}\right), q$ is a function on $R^{3}$, and $F$ is a real valued function of a real variable. Let $H=\mathscr{D}_{[\sqrt{-4}]} \oplus L_{2}$ where $\mathscr{D}_{[\sqrt{-4}]}$ is the completion of the domain of $\sqrt{-\Delta}$ in $L_{2}$ with respect to the norm $\|\nabla u\|_{2}=\left(\int_{R^{3}}|\nabla u|^{2}\right)^{1 / 2}$. The Sobolev inequality shows that we may treat the elements of $\mathscr{D}_{[\sqrt{-4}]}$ as functions. $\quad\left(\mathscr{D}_{[\sqrt{ }-4} \subset L_{6}\right)$. Let

$$
A=\left(\begin{array}{ll}
0 & I \\
\Delta & 0
\end{array}\right)
$$

on $H$, i.e., $A\left(\begin{array}{l}u \\ v\end{array}\right)=\left(\begin{array}{c}v \\ \Delta u\end{array}\right)$ for such $\left(\begin{array}{l}u \\ v\end{array}\right)$ where it is defined. $A$ is skew-adjoint with respect to $\left\|\left(\begin{array}{l}u \\ v\end{array}\right)\right\|^{2}=\|\nabla u\|_{2}^{2}+\|v\|_{2}^{2}$ where \|\|$_{2}$ is the $L_{2}$ norm. Let $K: H \rightarrow H$ be defined by $K\left(\begin{array}{l}u \\ v\end{array}\right)=\left(\begin{array}{c}0 \\ q F(u)\end{array}\right)$. If 
$r \in C_{0}^{\infty}$ and $s \in C_{0}^{\infty}$ then $u \in C_{0}^{\infty}$ for each $t$. Then $\left(\begin{array}{c}u(t) \\ u_{t}(t)\end{array}\right) \in H$ and

$$
\frac{d}{d t}\left(\begin{array}{c}
u \\
u_{t}
\end{array}\right)=A\left(\begin{array}{c}
u \\
u_{t}
\end{array}\right)+K\left(\begin{array}{c}
u \\
u_{t}
\end{array}\right)
$$

where $u$ satisfies equation (5). In general true solutions to

$$
\frac{d \alpha}{d t}=A \alpha+K \alpha
$$

are not strict solutions to (5) but are solutions in a weak sense. In fact however this paper will be interested in solutions to (6); i.e., mild solutions. For a discussion of the above see $[2,3,4,5]$.

Proposition 6. Assume $F(0)=0,|F(x)-F(y)|<k|x-y|$ and $|q(x)| \leqq \gamma e^{-c|x|},|x|=\left(x_{1}^{2}+x_{2}^{1}+x_{3}^{2}\right)^{1 / 2}$ where

$$
C>\left(\frac{2 k \gamma \pi^{1 / 3} d}{3}\right)^{1 / 2}
$$

$d=\sup _{u \in 0_{0}^{\infty}}\left(\|u\|_{6} /\|\nabla u\|_{2}\right) . \quad(d$ is finite by the Sobolev lemma). Then if $\varphi_{0} \in C_{0}^{\infty} \oplus C_{0}^{\infty} \subset H, \varphi_{0} \in \mathscr{R}_{-}$(and $\mathscr{R}_{-}$).

Proof.

$$
\|K(\rho)-K(\psi)\| \leqq \eta\|\varphi-\psi\| \quad \text { where } \eta=\frac{2 k \gamma \pi^{1 / 3} d}{3 c}
$$

Thus there exist unique solutions of (6) for any initial data. By Huygen's principle, $e^{t A} \varphi_{0}$ is detached; i.e., $\exists t_{0}$ such that if

$$
\left(\begin{array}{c}
u(t) \\
u_{t}(t)
\end{array}\right)=e^{t A} \varphi_{0}
$$

$u(t)$ and $u_{t}(t)$ vanishes in a cone $\left\{|x|<|t|+t_{0}\right\}$. Thus

$$
\begin{aligned}
\left\|K\left(e^{t A} \varphi_{0}\right)\right\|^{2} & =\int q^{2} F^{2}(u(t)) d_{3} x \leqq k^{2} \int q^{2} u^{2}=k^{2} \int_{|x| \geqq|t|+t_{0}} q^{2} u^{2} \\
& \leqq k^{2} d^{2}\left(\int_{|x| \geqq|t|+t_{0}} q^{3}\right)^{2 / 3}\left\|\varphi_{0}\right\|^{2} \\
& \leqq k^{2} d^{2}\left\|\varphi_{0}\right\|^{2} \gamma^{2} \frac{(4 \pi)^{2 / 3} e^{-2 c t_{0}}}{9 c^{2}} e^{-2(c-\varepsilon)|t|}
\end{aligned}
$$

for any $\varepsilon>0$ and $|t|>T(\varepsilon)$. Our result then follows from Proposition 4 .

REMARK. Note that only the fact that $u(t)$ is detached is used. 
Proposition 7. Assume $F(u)=u^{3}, \quad|q(x)| \leqq \gamma e^{-c|x|^{2}}$. Then if $\varphi_{0} \in C_{0}^{\infty} \oplus C_{0}^{\infty}, \varphi_{0} \in \mathscr{R}_{+}$and $\mathscr{R}_{-}$.

Proof. It is proved in Segal [2] that for this case, solutions exist for any initial data. $\|K(\alpha)-K(\beta)\| \leqq 3 \gamma d^{3} r^{2}\|\alpha-\beta\|$ where $\|\alpha\|$, $\|\beta\| \leqq r$, i.e, $k(s)$, is the notation of Proposition 5 , is $3 \gamma d^{3} r$ (use Sobolev inequality). Suppose $\varphi_{0} \in C_{0}^{\infty} \oplus C_{0}^{\infty}$. It will be shown that for $|t|$ sufficiently large

$$
\left\|K\left(e^{t A} \varphi_{0}\right)\right\| \leqq \gamma_{1} e^{-c^{\prime}|t|} \quad \text { where } \quad c^{\prime}>k\left(2\left\|\varphi_{0}\right\|\right) .
$$

The result then follows from Proposition 5. Let $\phi_{0}(t)=\left(\begin{array}{c}u(t) \\ u_{t}(t)\end{array}\right)$.

$$
\left\|K\left(e^{t A} \varphi_{0}\right)\right\|^{2}=\int q^{2} u^{6}
$$

But $u(t)$ is detached so

$$
\begin{aligned}
& \left\|K\left(e^{t A} \varphi_{0}\right)\right\|^{2}=\int_{|x| \geqq|t|+t_{0}} q^{2} u^{6} \leqq e^{-c\left(|t|+t_{0}\right)^{2}} \int_{|x| \geqq|t|+t_{0}} u^{6} \\
& \leqq e^{\left.-c|| t \mid+t_{0}\right)^{2}} d^{6}\left\|\varphi_{0}\right\|^{6} . \\
& \therefore \quad\left\|K\left(e^{t A} \varphi_{0}\right)\right\| \leqq d^{3}\left\|\varphi_{0}\right\|^{3} e^{-c\left(|t|+t_{0}\right)^{2}} .
\end{aligned}
$$

For $|t|$ sufficiently large

$$
e^{-c\left(|t|+t_{0}\right)^{2}} \leqq \gamma_{1}^{2} e^{-c^{\prime}|t|}
$$

for arbitrary $c^{\prime}$.

By Propositions 7 and 5 there exists a $\psi_{t}(t)$ satisfying (2) mildly such that

$$
\left\|\psi_{ \pm}(t)-e^{t A} \varphi_{0}\right\| \leqq \gamma e^{-c|t|} \quad|t| \leqq T(c)
$$

where $\varphi$ and $K$ satisfies the hypothesis of Proposition 7 where $c$ is arbitrary. Fix $c$. Then for $|t|$ sufficiently large, $\left\|\psi_{ \pm}\right\| \leqq 2\left\|\varphi_{0}\right\|$. Thus

$$
\begin{aligned}
\left\|K\left(\psi_{ \pm}(t)\right)\right\| & \leqq\left\|K\left(\psi_{ \pm}(t)\right)-K\left(e^{t A} \varphi_{0}\right)\right\|+\left\|K\left(e^{t A} \varphi_{0}\right)\right\| \\
& \leqq k\left(2\left\|\varphi_{0}\right\|\right) \gamma e^{-c|t|}+\gamma^{\prime} e^{-c|t|}=\gamma e^{-c|t|}
\end{aligned}
$$

i.e.

$$
\begin{aligned}
& \left\|K\left(\psi_{ \pm}(t)\right)\right\|<\gamma_{+} e^{-c t} \quad \text { for } \quad t \geqq T_{+}(c) \\
& \left\|K\left(\psi_{-}(t)\right)\right\|<\gamma_{-} e^{c t} \quad \text { for } \quad t \leqq T_{-}(c)
\end{aligned}
$$

for arbitrary positive $e$. Thus:

CoROllary. If $K$ is as in Proposition 7, there exist solutions to $(d \varphi / d t)=A \varphi+K(\varphi)$, satisfying $\left(^{*}\right)$ and $(* *)$.

REMARK 1. It is not clear whether there exist $\varphi$ satisfying $\left(^{*}\right)$ 
and $(* *)$ simultaneously.

REMARK 2. Strauss $[4,5]$ by using energy inequalities has shown for the case $F(u)=q u^{3}$ that there exist solutions $u(x, t)$ such that $\|K(u(t))\|$ are integrable which implies they are in the domain of $\mathscr{W}$ - by Proposition 1.

4. Nonlinear relativistic wave equation. Consider the partial differential equation

$$
\square u=m^{2} u+q F(u) \quad u(0)=r \quad u_{t}(0)=s
$$

where $m>0$. By a result of R. W. Goodman [1], has no finite energy detached solutions. As in $\S 3$, consider mild solutions to

$$
\frac{d \varphi}{d t}=A \varphi+K(\varphi) \quad \varphi(0)=\varphi_{0}
$$

where in this case $H=\mathscr{D}_{\left[\sqrt{m^{2}-4}\right]} \oplus L_{2}$ and

$$
\begin{gathered}
A=\left(\begin{array}{cc}
0 & I \\
-\left(m^{2}-\Delta\right) & 0
\end{array}\right) \\
\left\|\left(\begin{array}{l}
u \\
v
\end{array}\right)\right\|^{2}=\left\|\sqrt{m^{2}-\Delta} u\right\|_{2}^{2}+\|v\|_{2}^{2} .
\end{gathered}
$$

The following will be proved elsewhere (in a somewhat stronger form).

LEMma. Let $r(x)$ and $s(x)$ be in $L_{2}\left(R^{3}\right)$. Let $\hat{r}$ and $\hat{s}$ be their Fourier transforms. Assume $\left(m^{2}+|x|^{2}\right)^{2} \hat{r}$ and $\left(m^{2}+|x|^{2}\right)^{2} \hat{s}$ are $e_{1}^{\prime}$ in $\Gamma$ where $\Gamma$ is the image under Fourier transform of $L_{1}\left(R^{3}\right)$. Then $\|u(x, t)\|_{\infty}=0(1 /|t|)$ where $u(x, t)$ satisfies (4) with $F \equiv 0$.

Proposition 8. Suppose $q$ is bounded, $F$ Lipschitz and $F \equiv 0$ in a neighborhood of 0 . Then if the initial data satisfy the requirements of the above lemma $\left\|K\left(e^{t A} \varphi_{0}\right)\right\| \rightarrow 0$ exponentially. In fact $\left\|K\left(e^{t A} \varphi_{0}\right)\right\|=$ 0 for $|t|$ sufficiently large. Thus $\mathscr{W}_{ \pm}$have nonempty range by Proposition 4.

Proof. As before, if

$$
\varphi=\left(\begin{array}{l}
u \\
v
\end{array}\right) \in H, K(\varphi)=\left(\begin{array}{c}
0 \\
-q F(u)
\end{array}\right) .
$$

Let $\varphi_{0}=\left(\begin{array}{l}r \\ s\end{array}\right)$ where $r$ and $s$ satisfy the above lemma. Then 


$$
e^{t A} \varphi_{0}=\left(\begin{array}{c}
u(x, t) \\
u_{t}(x, t)
\end{array}\right)
$$

Thus

$$
K\left(e^{t A} \varphi_{0}\right)=\left(\begin{array}{c}
0 \\
-q F(u(x, t))
\end{array}\right) .
$$

But there exists $\varepsilon>0$ such that if $|y|<\varepsilon, F(y)=0$ by hypothesis. By the above lemma, there exists a $T$ such that if $|t|>T$, $\|u(x, t)\|_{\infty}<\varepsilon$. Thus for $|t|>T, F(u(x, t))=0$ for all $x \in R^{3}$. If $F$ is Lipschitz continuous, so then is $K$.

I. E. Segal has recently obtained by similar methods stronger results along the above lines.

Added in proof. The hypothesis of Proposition 4 and 5 may be weakened to $\int_{-\infty}^{0} e^{-c t}\left\|K\left(e^{t A} \beta\right)\right\| d t<\infty$ for some $c>k$.

\section{BIBLIOGRAPHY}

1. R. W. Goodman, One sided invariant subspaces and domain of uniqueness for hyperbolic equations, Proc. Amer. Math. Soc. 15 (1964), 653.

2. I. E. Segal, Non-linear semi-groups, Ann. of Math. 78 (1963), 339-364.

3. - Differential operators in the manifold of Solutions of a non-linear differential equation, J. Math. Pures Appl. 44 (1965), 71-105.

4. W. Strauss, La decroisance asymptotique des solutions des equations d'onde nonlineaires, Comptes Rendus 256 (1963), 2749-2750.

5. - Les operators d'onde pour des equations d'onde non-lineaires independantes du temps, Comptes Rendus 256 (1963), 5045-5046.

6. - Scattering for hyperbolic equations, Trans. Amer. Math. Soc. 108 (1963), $13-37$.

Received July 15, 1965. Portions of this paper were contained in the author's doctoral dissertation directed by Professor I. E. Segal and presented to the Massachusetts Institute of Technology. 


\section{PACIFIC JOURNAL OF MATHEMATICS}

\section{EDITORS}

\author{
H. SAMELSON \\ Stanford University \\ Stanford, California \\ J. P. JANS \\ University of Washington \\ Seattle, Washington 98105
}

\author{
J. DUGUNDJI \\ University of Southern California \\ Los Angeles, California 90007 \\ RICHARD ARENS \\ University of California \\ Los Angeles, California 90024
}

\section{ASSOCIATE EDITORS}
E. F. BECKENBACH
B. H. NeumanN
F. WOLF
K. YOSIDA

\section{SUPPORTING INSTITUTIONS}

\author{
UNIVERSITY OF BRITISH COLUMBIA \\ CALIFORNIA INSTITUTE OF TECHNOLOGY \\ UNIVERSITY OF CALIFORNIA \\ MONTANA STATE UNIVERSITY \\ UNIVERSITY OF NEVADA \\ NEW MEXICO STATE UNIVERSITY \\ OREGON STATE UNIVERSITY \\ UNIVERSITY OF OREGON \\ OSAKA UNIVERSITY \\ UNIVERSITY OF SOUTHERN CALIFORNIA
}

STANFORD UNIVERSITY

UNIVERSITY OF TOKYO

UNIVERSITY OF UTAH

WASHINGTON STATE UNIVERSITY

UNIVERSITY OF WASHINGTON

AMERICAN MATHEMATICAL SOCIETY CHEVRON RESEARCH CORPORATION TRW SYSTEMS

NAVAL ORDNANCE TEST STATION 


\section{Pacific Journal of Mathematics}

\section{Vol. 19, No. 1 \\ May, 1966}

A. R. Brodsky, The existence of wave operators for nonlinear equations... 1

Gulbank D. Chakerian, Sets of constant width................... 13

Robert Ray Colby, On indecomposable modules over rings with minimum condition....................................... 23

James Robert Dorroh, Contraction semi-groups in a function space ....... 35

Victor A. Dulock and Harold V. McIntosh, On the degeneracy of the Kepler



James Arthur Dyer, The inversion of a class of linear operators ......... 57

N. S. Gopalakrishnan and Ramaiyengar Sridharan, Homological dimension of Ore-extensions ................................. 67

Daniel E. Gorenstein, On a theorem of Philip Hall ................. 77

Stanley P. Gudder, Uniqueness and existence properties of bounded observables..................................... 81

Ronald Joseph Miech, An asymptotic property of the Euler function ....... 95

Peter Alexander Rejto, On the essential spectrum of the hydrogen energy and related operators ............................... 109

Duane Sather, Maximum and monotonicity properties of initial boundary



Peggy Strait, Sample function regularity for Gaussian processes with the parameter in a Hilbert space ........................... 159

Donald Reginald Traylor, Metrizability in normal Moore spaces ........... 175

Uppuluri V. Ramamohana Rao, On a stronger version of Wallis' formula ...............................

Adil Mohamed Yaqub, Some classes of ring-logics....... 